\title{
Improved preservation of function during acoustic neuroma surgery
}

\author{
*Hirofumi Nakatomi, MD, PhD, ${ }^{1}$ Hidemi Miyazaki, MD, ${ }^{2}$ Minoru Tanaka, MD, PhD, ${ }^{1}$ \\ Taichi Kin, MD, PhD, ${ }^{1}$ Masanori Yoshino, MD, ${ }^{1}$ Hiroshi Oyama, MD, PhD, ${ }^{3}$ Masaaki Usui, MD, PhD, ${ }^{4}$ \\ Hiroshi Moriyama, MD, PhD, ${ }^{2}$ Hiromi Kojima, MD, PhD, ${ }^{2}$ Kimitaka Kaga, MD, PhD, ${ }^{5}$ \\ and Nobuhito Saito, MD, PhD'
}

\begin{abstract}
1Department of Neurosurgery, University of Tokyo Hospital; '2Department of Otolaryngology, Jikei University; ${ }^{3}$ Department of Medical Informatics, University of Tokyo; ${ }^{4}$ Department of Neurosurgery, Toranomon Hospital; and ${ }^{5}$ Department of Otolaryngology, Tokyo Medical Center, Tokyo, Japan
\end{abstract}

\begin{abstract}
OBJECT Restoration of cranial nerve functions during acoustic neuroma (AN) surgery is crucial for good outcome. The effects of minimizing the injury period and maximizing the recuperation period were investigated in 89 patients who consecutively underwent retrosigmoid unilateral AN surgery.
\end{abstract}

METHODS Cochlear nerve and facial nerve functions were evaluated during AN surgery by use of continuous auditory evoked dorsal cochlear nucleus action potential monitoring and facial nerve root exit zone-elicited compound muscle action potential monitoring, respectively. Factors affecting preservation of function at the same (preoperative) grade were analyzed.

RESULTS A total of 23 patients underwent standard treatment and investigation of the monitoring threshold for preservation of function; another 66 patients underwent extended recuperation treatment and assessment of its effect on recovery of nerve function. Both types of final action potential monitoring response and extended recuperation treatment were associated with preservation of function at the same grade.

CONCLUSIONS Preservation of function was significantly better for patients who received extended recuperation treatment.

http://thejns.org/doi/abs/10.3171/2014.8.JNS132525

KEY WORDS acoustic neuroma surgery; continuous direct brainstem auditory evoked potential monitoring; oncology

A COUSTIC neuromas (ANs), also called vestibular schwannomas, are benign Schwann cell tumors that usually arise within the internal auditory canal from the superior vestibular nerve or inferior vestibular nerve. ${ }^{19}$ The therapeutic goal of AN surgery is total tumor excision, with preservation of facial nerve and hearing function. However, for nearly $50 \%$ of patients with large or adhesive ANs, surgery results in a poor grade of facial nerve function, such as House-Brackmann (HB) Grade II or higher, and a worse grade of hearing according to the Gardner-Robertson (GR) scale..$^{13,18,19}$ Such poor preserva- tion of function after the conventional surgical strategy is related to the various aspects of the nerves, tumors, and neurosurgeons.

Intraoperative monitoring of the facial nerve $\mathrm{e}^{7,26,27,30}$ and the cochlear nerve is essential for improved preservation of function after surgery, but conventional monitoring methods have proven inadequate..$^{8,14,15,20,28}$ Therefore, novel methods (continuous direct neurophysiological monitoring) have been introduced to achieve immediate intraoperative feedback and successful prognostic assessment..$^{2,6,10-12,16,17,21,25}$ Two methods that have the potential to

ABBREVIATIONS AEDNAP = auditory evoked dorsal cochlear nucleus action potential; $A N=$ acoustic neuroma; $A P R=$ amplitude preservation ratio; $A U C=$ area under the curve; BAEP = brainstem auditory-evoked potential; FREMAP = facial nerve root exit zone-elicited compound muscle action potential; GR = Gardner-Robertson; HB = House-Brackmann; M-max = maximum peak-to-peak amplitude; $R O C=$ receiver operating characteristic.

SUBMITTED December 10, 2013. ACCEPTED August 26, 2014.

INCLUDE WHEN CITING Published online October 24, 2014; DOI: 10.3171/2014.8.JNS132525.

DISCLOSURE This study was supported by the Ministry of Education, Culture, Sports, Science and Technology/Japan Society for the Promotion of Science Grant-in-Aid for Scientific Research (no. 24592154) and by the Japan Brain Foundation Grant-in-Aid for Scientific Research 2012. The authors report no conflict of interest concerning the materials or methods used in this study or the findings specified in this paper.

* Drs. Nakatomi and Miyazaki contributed equally to this work. 
warn neurosurgeons of neural damage directly caused by surgical procedures are continuous direct auditory evoked dorsal cochlear nucleus action potential (AEDNAP) monitoring and facial nerve root exit zone-elicited compound muscle action potential (FREMAP) monitoring. Fortunately, gradual reversal of cochlear nerve injury after surgical procedures has been confirmed in humans ${ }^{25,26}$ and animals. ${ }^{1,3-5,9,22-24,29}$

The study reported here tested our hypothesis that nerve function in adults could be restored after iatrogenic damage by minimizing the injury period and maximizing the recuperation period during surgery, which could help limit irreversible injury and maximize preservation of function. The mechanisms of neural injuries were classified as traction, crushing, thermal insult, and ultrasonic injury. Changes in nerve function were evaluated by 3 parameters: acute decline of function immediately after surgical injury (hereafter called acute decline), recuperation time during which surgical procedures were temporarily stopped to allow functional recovery (recuperation time), and permanent loss of function after maximum recovery (permanent loss).

\section{Methods}

\section{Patients}

This study included 89 consecutively enrolled patients with a newly diagnosed AN treated with retrosigmoid surgery and followed up at the University of Tokyo Hospital (Tokyo, Japan) from 2006 through 2011. All patients had a histologically confirmed AN (vestibular schwannoma) and were 15 years of age or older. The study excluded 26 patients who had neurofibromatosis Type 2 or recurrent tumor or who had previously undergone radiation therapy. Gross-total or near-total resection was performed, if feasible. Tumor removal was classified as total, near total $(\geq$ $98 \%)$, subtotal $(\geq 95 \%)$, or partial $(<95 \%)$ according to the findings on postoperative MR images. ${ }^{19}$ All patients underwent retrosigmoid craniotomy and tumor resection under continuous direct AEDNAP and FREMAP monitoring (performed by H.N.). On postoperative Days 1-7, all patients received intravenous dextran and corticosteroids.

\section{Surgery-Related Response Change}

Among the typical intradural procedures that had the potential to affect nerve function, the following 10 steps were identified: 1) opening the foramen magnum during drainage of cerebrospinal fluid, 2) cerebellar retraction and insertion of electrodes, 3) coring, 4) partial V-shaped resection, 5) shaving along the proximal facial nerve, 6) shaving along the proximal cochlear nerve, 7) internal auditory canal drilling, 8) shaving along the distal facial nerve, 9) shaving along the distal cochlear nerve, and 10) removal of the fundal tumor. Any changes in the continuous AEDNAP and FREMAP monitoring responses were recorded by using the 3 parameters: acute decline, recuperation time, and permanent loss.

\section{Monitoring and Microanatomy of Brainstem Relationships}

Continuous Direct AEDNAP Monitoring

After undergoing intubation and induction of general anesthesia, patients were prepared for monitoring of cranial nerve VIII by use of the conventional brainstem auditory evoked potential (BAEP) method (Fig. 1A and D). Sevoflurane was used only for initial induction; intravenous propofol was used as the main anesthetic agent. Under bispectral index monitoring, target control infusion of propofol was performed as the drug concentration in the brain was maintained at $3.0 \mu \mathrm{g} / \mathrm{ml}$. The electrode for AEDNAP monitoring (Ad-Tech Medical Instrument Corporation) was placed on the dorsal cochlear nucleus at the bottom of the gap between the middle cerebellar peduncle and the floor of the fourth ventricle instead of on the auricle. The tela choroidea membrane pouching from the fourth ventricle just beneath the choroid plexus and just behind the glossopharyngeal-vagal nerve complex was sharply cut and opened to form a clear window into the lateral recess. The AEDNAP electrode was inserted through the lateral recess along the glossopharyngeal nerve by moving the cerebellum medially and posteriorly and was positioned on the surface of the dorsal cochlear nucleus (Fig. 1B, C, and E). The amplitude preservation ratio (APR) of the AEDNAP response (defined as the base-to-peak amplitude of the current AEDNAP response divided by the starting AEDNAP response, multiplied by 100) was calculated throughout the procedure and displayed as a trend graph, which was used to track cochlear nerve function (Fig. 1F-H). The final AEDNAP APR was also defined as the base-to-peak amplitude of the AEDNAP response before dural closure divided by the starting AEDNAP response and multiplied by 100 . Before closure of the dura, the AEDNAP electrode was removed by gentle traction on the lead wire.

\section{Continuous Direct FREMAP Monitoring}

For FREMAP monitoring, the facial nerve root at the brainstem was electrically stimulated $(3 \mathrm{~Hz})$. The compound muscle action potential elicited by stimulation of the facial nerve root entry zone was continuously monitored by using the Neuropack MEE2000 recording system (Nihon Kohden). Except for use of a short-acting induction agent (rocuronium bromide up to $0.9 \mathrm{mg} /$ $\mathrm{kg}$ of body weight) for the initial 30 minutes, paralytic agents were strictly avoided throughout the operation. Recording and stimulation conditions were the same as for conventional intermittent direct facial electromyographic recording (Fig. 2A and B). A specially designed hat-shaped monopolar electrode (Ad-Tech Medical Instrument Corporation), called the FREMAP electrode, was placed on the facial nerve root entry zone medial to the glossopharyngeal nerve (Fig. 2C-E). The maximum peak-to-peak amplitude (M-max, in mV) was measured for each muscle by increasing the electrical stimulus intensity. To evoke stable maximum muscle action potential amplitudes, the lowest electrical stimulation $(0.2-2.0 \mathrm{~mA})$ was used to initiate continuous FREMAP monitoring (Fig. 2D). Throughout the procedure, the APR of the M-max (current M-max divided by the starting M-max, multiplied by 100) was calculated and plotted as a trend graph to visualize and track the function of the facial nerve near the orbicularis oculi and orbicularis oris muscles (Fig. 2F-H). The final orbicularis oris muscle FREMAP APR was defined as the base-to-peak amplitude of the FREMAP response before 

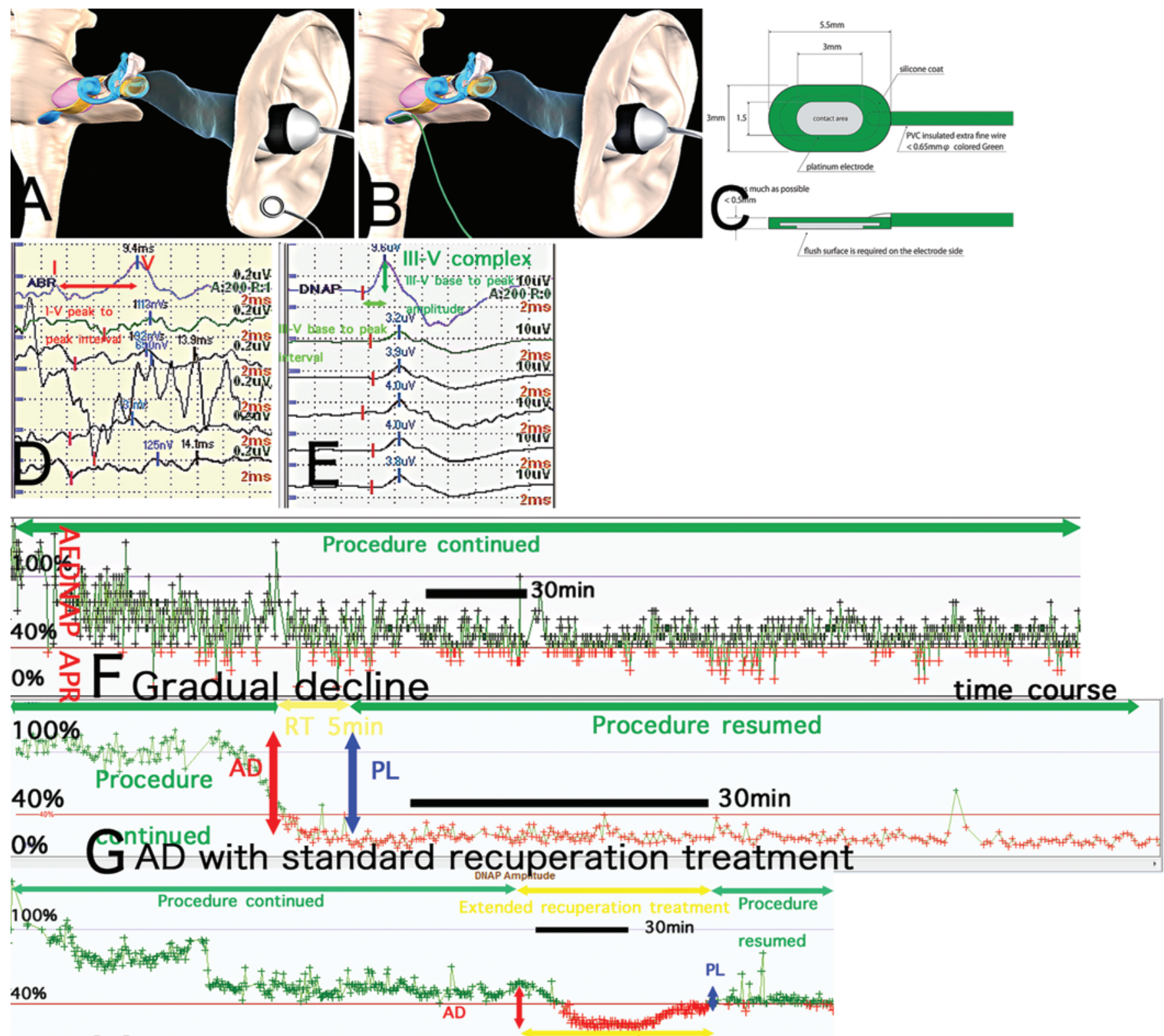

\% 1 AD with extended reccrperation treatment

FIG. 1. BAEP and AEDNAP monitoring. BAEP monitoring records potentials from the auricle (A), whereas AEDNAP records direct nuclear compound action potentials from the dorsal cochlear nucleus (B). The AEDNAP electrode was specifically designed to fit to the dorsal cochlear nucleus (C). AEDNAP monitoring detected large-scale uniform waveforms corresponding to auditory brainstem response III, IV, and $\mathrm{V}$ wave complexes $(\mathrm{E})$. These waveforms were stable, and the potentials were 20 - to 30 -fold higher $(10-20 \mu \mathrm{V})$ than conventionally measured BAEP waves $(0.2-1 \mu \mathrm{V})(\mathrm{D})$. AEDNAP trend graphs from 3 operative scenarios and outcomes are shown. For this patient (F), decline was gradual and the final APR was $45 \%$ and hearing was preserved at the same grade. For this patient (G), acute decline (from $100 \%$ to 10\%) caused by crush injury was followed by 5 minutes of recuperation time, resulting in final APR of 10\%; permanent loss was similar to the acute decline (reduced hearing grade from GR Grade II to Grade V). For this patient (H), acute decline (from $60 \%$ to $20 \%$ ) caused by crush injury was followed by 45 minutes of recuperation time; enhanced recovery resulted in a final APR of $42 \%$, permanent loss of $60 \%$ to $42 \%$ (much smaller than the acute decline), and preserved same-grade hearing. Preservation of function rate was significantly better for patients who received extended recuperation time $(\mathbf{H})$ than for those who received conventional recuperation time $(G)$. $A D=$ acute decline; $P L=$ permanent loss.

dural closure divided by the starting FREMAP response, multiplied by 100 .

All protocols were approved by the Institutional Review Board and the Ethics Committee of Tokyo University Hospital.

\section{Standard Treatment Versus Extended Recuperation Treatment}

The AEDNAP and FREMAP waveforms were monitored during each of the 8 procedures performed during Steps $3-10$, every $5-15$ seconds for the cochlear nerve 

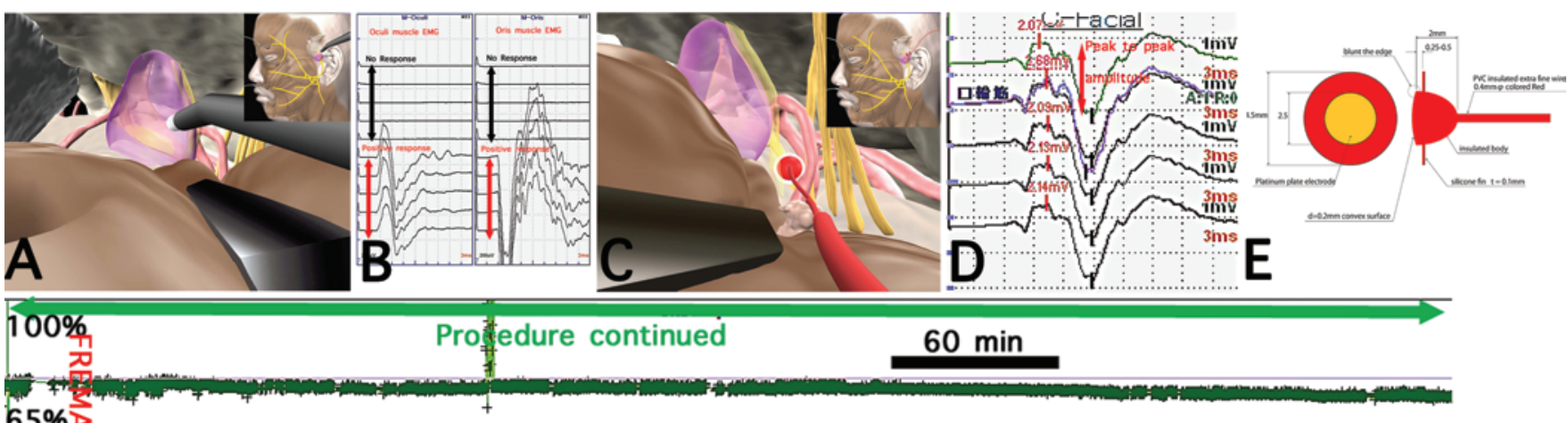

$65 \% \gg$

\% $\frac{?}{0}$ F Gradual decline

time course

Procedure
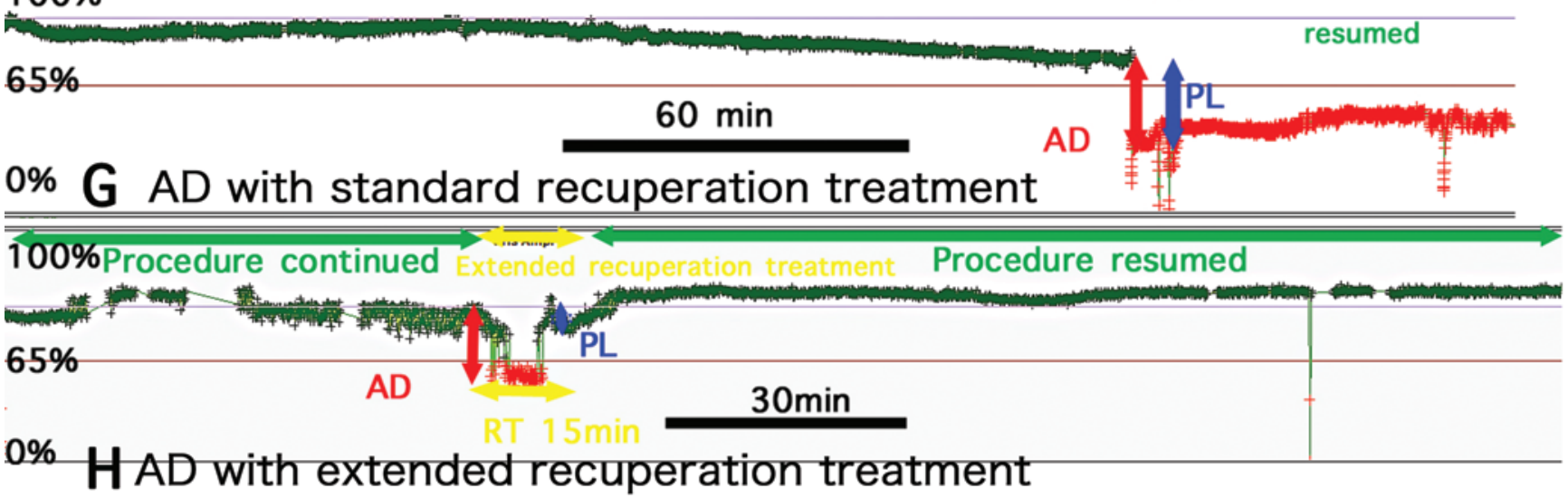

FIG. 2. Conventional intermittent stimulation and FREMAP monitoring. Conventional recording intermittently stimulates the facial nerve fascicles on the tumor capsule to evoke muscle action potentials (A) to determine whether the stimulation point is located close to or on the facial nerve fascicles at discrete time points (B). FREMAP continuously measures potentials every 3 seconds (C), resulting in maximal compound action potentials from both the orbicularis oculi muscle and the orbicularis oris muscle (D). The FREMAP electrode was specifically designed to fit to the facial nerve root (E). Inset illustrations show the relationships among the facial muscle, facial nerve, tumor, and electrodes (A and C). For visualization and tracking of facial nerve function in the vicinity of the orbicularis oculi muscle and the orbicularis oris muscle, the APR was calculated and plotted as a trend graph (F-H). FREMAP trend graphs from 3 operative scenarios and outcomes are shown. For this patient $(F)$, decline was gradual, final FREMAP APR was $85 \%$, and facial nerve function was preserved at the same grade. For this patient (G), acute decline from $80 \%$ to $30 \%$, caused by crush injury, was followed by 5 minutes of recuperation time, resulting in a final APR of $40 \%$, permanent loss similar to the acute decline, and reduced facial nerve function from HB Grade I to Grade II. For this patient $(\mathrm{H})$, acute decline from $90 \%$ to $40 \%$, caused by crush injury, was followed by 15 minutes of recuperation time with enhanced recovery, resulting in a final APR of $85 \%$, permanent loss of $90 \%$ to $85 \%$ (much smaller than the acute decline), and preservation of facial nerve function at the same grade.

and every 3 seconds for the facial nerve. Over the study period, our strategy evolved to focus on the recuperation treatment, resulting in a standard treatment group of 23 patients (who underwent treatment during 2006-June 2008) and an extended recuperation treatment group of 66 patients (who underwent treatment during July 20082011) (Figs. $1 \mathrm{~F}-\mathrm{H}$ and $2 \mathrm{~F}-\mathrm{H}$ ). Patients in the standard treatment group underwent treatment according to the previous protocol, in which after a change in the monitoring response (acute decline) was detected, the neurosurgeon temporarily stopped the surgical procedure to allow neurological recovery for a period (recuperation time) that ranged from a few seconds to several minutes, depending on the neurosurgeon's intuition and experience.
Therefore, we investigated the monitoring thresholds for preservation of same (preoperative)-grade function in this standard treatment group. After specific threshold values for recovery of the facial and cochlear nerves were obtained for patients in the standard treatment group, we investigated whether extended recuperation treatment could restore better nerve function than the standard treatment. For patients in the extended recuperation treatment group, the protocol required recuperation time to be extended every 15 minutes until these responses returned to the respective threshold values and final permanent loss was measured. Therefore, we could discriminate between irreversible and reversible events caused by specific surgical procedures. 


\section{Follow-Up Evaluations and Outcomes}

Contrast-enhanced MRI was performed every 6 months for the 1st postoperative year and annually thereafter. Facial nerve function was evaluated by using the HB grading system ${ }^{7}$ within 3 weeks of surgery and categorized as HB Grade I (no facial nerve palsy), HB Grade II (slight facial nerve palsy), or HB Grades III-VI (severe facial nerve palsy). We recorded the worst HB grade within 3 weeks after surgery. Hearing function was assessed by using the GR grading system,${ }^{19}$ and we recorded the worst GR grade within 3 weeks of surgery.

\section{Statistical Analysis}

For patients in the standard treatment group, specific threshold values were identified by evaluating the area under the curve (AUC) of the receiver operating characteristics (ROC) curve. The Youden index J was used as the criterion for identifying the most appropriate cutoff point, for which $\{\mathrm{Se}(\mathrm{c})+\mathrm{Sp}(\mathrm{c})-1\}$, in which $\mathrm{Se}(\mathrm{c})$ indicates sensitivity and $\mathrm{Sp}(\mathrm{c})$ indicates specificity, was maximized and ranged between 0 and 1 .

Forward and backward stepwise logistic regression analyses were used to determine associations of potential confounders with preservation of cochlear and facial nerve function: patient age, patient sex, tumor side, vestibular symptom duration, cochlear symptom duration, treatment group, extent of resection, tumor origin, degree of fanning, degree of meatal filling, tumor size in the internal auditory canal, and tumor size in the cerebellopontine angle. Additional cochlear nerve confounders were cochlear nerve route, cochlear nerve adhesion, and final AEDNAP APR. Additional facial nerve confounders were facial nerve route, facial nerve adhesion, initial stimulation, final stimulation, and final orbicularis oris muscle FREMAP APR. Nerve-specific mechanisms of surgical injury (traction, crushing, ultrasonic, and thermal) were evaluated by permanent loss-adjusted means for acute decline because the permanent loss might be affected by the acute decline. We used 1-way analysis of covariance with differences between the 2 groups as the dependent variable, permanent loss as the independent variable, and acute decline as the covariate.

Values for patient characteristics are presented as mean $\pm \mathrm{SD}$. Independent t-tests were used to analyze the data. Frequencies were compared by using the chi-square test or in subgroups with low sample size by using the Fisher's exact test to detect differences in proportions between the categorical variables of the 2 groups. A p value less than 0.05 was considered significant. SPSS Statistics software, version 19 (IBM), was used for analysis.

\section{Results}

\section{AEDMAP and FREMAP Monitoring}

\section{AEDNAP Monitoring of the Cochlear Nerve}

Conventional BAEP monitoring measures indirect field potentials from the auricle (Fig. 1A), whereas AEDNAP measures direct nuclear compound action potentials from the dorsal cochlear nucleus (Fig. 1B). The AEDNAP III$\mathrm{V}$ wave complex potentials had magnitudes of 3.0-4.0 $\mu \mathrm{V}$ (Fig. 1E), which are 20-30-fold higher than those of BAEP waves (Fig. 1D). One unit of the AEDNAP scale represents $10 \mu \mathrm{V}, 50$-fold higher than that of the BAEP scale. The changes in the peak latency and base-to-peak amplitude of this single complex waveform were investigated at various procedural stages (Fig. 1E). Of the 89 patients with unilateral AN, hearing was GR Grade I-III for 75 patients and Grade IV or V for 14 patients. The patients with GR Grade IV or V hearing were excluded from AEDNAP monitoring; all patients with GR Grade I-III hearing exhibited stable AEDNAP recordings at the start of surgery and were included in this analysis. Patients who underwent intraoperative AEDNAP monitoring could be assigned to 1 of 3 patterns: gradual decline, acute decline with standard treatment, and acute decline with extended recuperation treatment (Fig. $1 \mathrm{~F}-\mathrm{H})$.

\section{FREMAP Monitoring of the Facial Nerve}

Instead of recording muscle action potentials by using intermittent probe stimulation (Fig. 2A and B), FREMAP monitoring measures muscle action potentials evoked directly at the facial nerve root (Fig. 2C-E). We investigated the peak-to-peak amplitude changes at various stages of surgery. Facial nerve function was normal (HB Grade I) for all patients except for 5, whose preoperative facial nerve function was HB Grade II. At the start of surgery, FREMAP recordings were stable for all patients, but during surgery, FREMAP monitoring showed 3 patterns (Fig. $2 \mathrm{~F}-\mathrm{H})$.

\section{Specific Patterns of AEDNAP and FREMAP Changes}

AEDNAP and FREMAP monitoring of the cochlear and facial nerves did not seem compromised by differences in tumor size (Fig. 3A and C), nerve fanning (Fig. 3B and D), and nerve course (Fig. 3B and D). AEDNAP and FREMAP monitoring identified significant acute decline immediately after 12 specific procedures for the cochlear nerve and 10 procedures for the facial nerve, which were subcategorized according to the mechanism of action as traction injury caused by pulling (Fig. 3E and F), crush injury caused by direct nerve manipulation (Fig. 3G and $\mathrm{H}$ ), thermal injury caused by bipolar coagulation (Fig. 3I and $\mathrm{J}$ ), and ultrasonic injury caused by ultrasonic aspiration (Fig. 3K and L). Procedures associated with drilling were classified as thermal injury because prolonged manipulation and insufficient cooling are known to elevate temperature in situ. ${ }^{1,3,5}$ No ischemic injury was identified.

Traction injury of the cochlear nerve was caused during performance of the following 6 surgical procedures: Step 2 , traction medially at the V-zone; Step 2, traction laterally at the V-zone; Step 4, traction laterally at the V-zone; Step 8 , traction caudally at the porus; Step 9, traction caudorostrally at the porus; and Step 10, traction medially at the fundus. Crush injury of the cochlear nerve was caused during performance of the following 2 surgical procedures: Step 7, dissection at the internal auditory canal; and Step 9, irritation at the porus. Thermal injury of the cochlear nerve was caused during performance of the following 3 surgical procedures: Step 6, coagulation near the V-zone; Step 7, drilling within the internal auditory canal; and Step 9, coagulation at the porus. Ultrasonic injury of the cochlear 

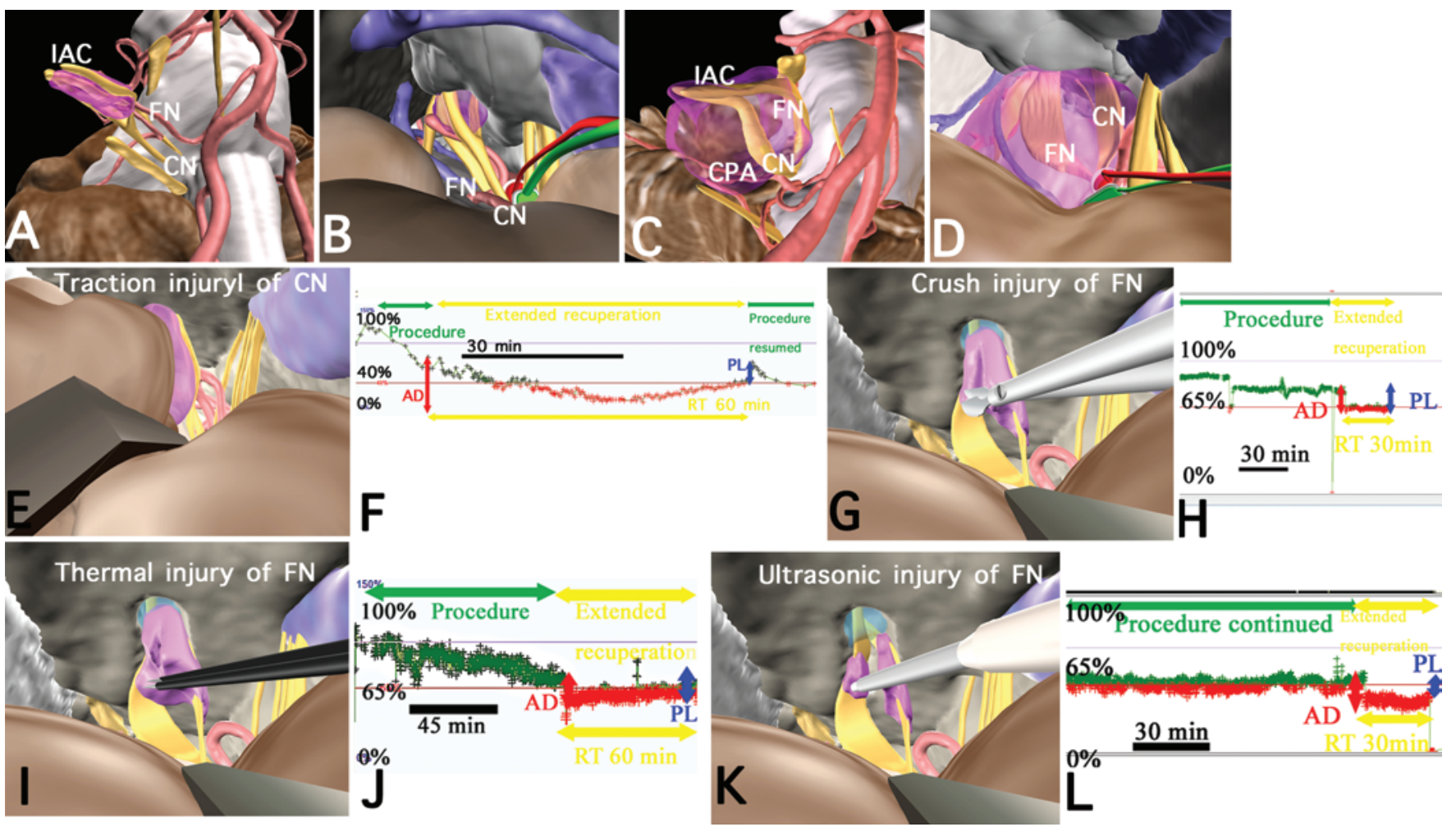

FIG. 3. Tumor, surgical, and monitoring factors. 3D anatomy of small ( $A$ and $B$ ) and large (C and D) ANs, shown by the anteroposterior views ( $A$ and $C$ ) and the intraoperative views on the right through retrosigmoid surgery ( $B$ and $D)$. Smaller tumor resides within the internal auditory canal (IAC), with dorsal displacement of the cochlear nerve (CN), nearly normal alignment of the facial nerve (FN), and little fanning of either nerve (A and B). Larger tumor extends into the cerebellopontine angle (CPA), with obvious rostromedial displacement and fanning of the facial nerve and ventral displacement and fanning of the cochlear nerve (C and D). The degree of meatal filling was around $90 \%$ in both nerves. AEDNAP (green) and FREMAP (red) electrodes could be safely positioned regardless of tumor size. APR trend graphs identified 4 types of nerve injury, and respective 3D illustrations represent the specific surgical procedure that caused the acute decline: traction injury caused by pulling of the cochlear nerve after cerebellar retraction ( $E$ and $F)$, crush injury caused by direct nerve manipulation of the facial nerve by microscissors $(G$ and $H)$, thermal injury caused by bipolar coagulation close to the facial nerve (I and J), and ultrasonic injury caused by ultrasonic aspiration close to the facial nerve ( $\mathrm{K}$ and $\mathrm{L})$. Ultrasonic injury is a direct neurological insult, but it is less traumatic than crush or thermal injury. Ultrasonic injury of the facial nerve seemed to respond to recuperation treatment. In contrast, ultrasonic injury of the cochlear nerve was severe. Avoiding severe injuries such as crush or thermal injury is essential, but extended recuperation treatment could help relieve nerve damage, especially after traction injury.

nerve was caused during performance of 1 procedure: Step 9, ultrasonic aspiration at the porus.

Traction injury of the facial nerve was caused during performance of the following 5 surgical procedures: Step 4 , traction rostrally at the V-zone; Step 5, traction rostrally at the root entry zone; Step 5, traction caudo-laterally at the V-zone; Step 8, traction laterally at the porus acusticus internus; and Step 10, traction medially at the fundus. Crush injury of the facial nerve was caused during performance of the following 2 surgical procedures: Step 4, dissection at the V-zone; and Step 8, irritation at the porus. Thermal injury of the facial nerve was caused during performance of 1 surgical procedure: Step 5, coagulation near the V-zone. Ultrasonic injury of the facial nerve was caused during performance of the following 2 procedures: Step 5, ultrasonic aspiration at the root entry zone; and Step 8, ultrasonic irritation at the porus.

\section{Neural Threshold for Optimal Preservation of Function}

For the 18 patients in the standard treatment group for cochlear nerve, analysis of ROC curve showed that the most optimal cutoff value of the final AEDNAP APR was $36.5 \%$ (AUC $=0.792 ; p=0.080 ;$ Youden index $J=0.683$ ). Logistic regression revealed that a final AEDNAP APR of 36.5\% or more predicted same-grade cochlear nerve preservation with an accuracy of $89.5 \%\left(p=0.005\right.$, Nagelkerke $R^{2}=$ $0.519)$. For the 23 patients in the standard treatment group for facial nerve, analysis of the ROC curve showed that the most optimal cutoff value of the final orbicularis oris muscle FREMAP APR was 61.5\% (AUC $=0.991 ; p=0.00023$; Youden index $\mathrm{J}=0.938)$. Logistic regression showed that a final orbicularis oris muscle FREMAP APR of $61.5 \%$ or more predicted same-grade facial nerve preservation with an accuracy of $95.7 \%\left(\mathrm{p}<0.0001\right.$, Nagelkerke $\left.\mathrm{R}^{2}=0.876\right)$. Acute decline below these thresholds (AEDNAP APR $36.5 \%$ and FREMAP APR 61.5\%) was addressed with further recuperation treatment and measurement of the final permanent loss as an indicator of neural damage. Thus, whenever an AEDNAP APR below 40\% and FREMAP APR below $65 \%$ were observed, the trend graphs notified the surgeons that neural damage was progressing. To facilitate visual recognition, on the monitoring screen the color 
of response below these warning levels turned from green to red (Figs. 1F-H, 2F-H, 3F, H, J, and L). Associations with postoperative hearing and facial function were then studied.

\section{Enhanced Recovery After Injury by Extended Recuperation Treatment}

Table 1 summarizes the characteristics of the patients, tumors, and other clinical factors. When these characteristics were compared between the 2 treatment groups, no significant differences were observed

\section{Rates of Nerve Preservation and Prognostic Predictors}

Of the 75 patients with GR Grade I-III hearing, samegrade hearing was preserved for $42(73.7 \%)$ of 57 patients in the extended recuperation treatment group and for 5 (27.8\%) of 18 patients in the standard treatment group. Multivariate logistic regression analysis of these 75 patients demonstrated that significant prognostic predictors for same-grade hearing preservation were final AEDNAP APR (adjusted OR in the extended recuperation treatment group relative to the standard treatment group, $0.940 ; 95 \%$ CI $0.909-0.973 ; \mathrm{p}<0.0001$ ) and treatment group (adjusted OR 0.177; 95\% CI 0.042-0.752; $\mathrm{p}=0.019$ ) (Table 2).

Of the 89 patients with preoperative HB Grade I-II, normal facial nerve function (HB Grade I) was achieved within 3 weeks after surgery for $54(81.8 \%)$ of 66 patients in the extended recuperation treatment group and in 7 (30.4\%) of 23 patients in the standard treatment group. Multivariate logistic regression analysis of these 89 patients demonstrated that significant prognostic predictors for same-grade facial nerve preservation were final orbicularis oris muscle FREMAP APR (adjusted OR in the extended recuperation treatment group relative to the standard treatment group, 0.910; 95\% CI 0.871-0.951; $\mathrm{p}<$ 0.0001 ) and treatment group (adjusted OR 0.186; 95\% CI 0.045-0.772; $\mathrm{p}=0.021$ ) (Table 2).

Final AEDNAP APR and final orbicularis oris muscle FREMAP APR were significantly associated with same-grade preservation of function, which was significantly better for the 39 patients with final AEDNAP APR of $36.5 \%$ or more than for the 36 patients with final AEDNAP APR less than 36.5\% (chi-square test, $\mathrm{p}<$ $0.0001, \eta=0.583$ ). Same-grade preservation of function was significantly greater for the 61 patients with a final orbicularis oris muscle FREMAP APR of $61.5 \%$ or more than for the 28 patients with a final orbicularis oris muscle FREMAP APR less than $61.5 \%$ (chi-square test, $\mathrm{p}<$ $0.0001, \eta=0.687$ ).

\section{Permanent Loss Changes and Surgical Procedures}

Table 3 summarizes the characteristics of monitor-

TABLE 1. Patient and tumor characteristics

\begin{tabular}{|c|c|c|c|}
\hline \multirow[b]{2}{*}{ Characteristic } & \multicolumn{2}{|c|}{ Treatment Group } & \multirow[b]{2}{*}{ p Value } \\
\hline & Standard $(n=23)$ & Extended Recuperation $(n=66)$ & \\
\hline Age in yrs* & $54.1 \pm 12.4$ & $48.6 \pm 13.9$ & 0.092 \\
\hline $\mathrm{M} / \mathrm{F}$ ratio & $11 / 12$ & $33 / 33$ & 0.86 \\
\hline Side, rt/lt & $11 / 12$ & $33 / 33$ & 0.86 \\
\hline Tumor origin, SVN/IVN & $8 / 15$ & $15 / 51$ & 0.26 \\
\hline FN route, com vent/rost/caud & $14 / 9 / 0$ & $42 / 21 / 3$ & 0.51 \\
\hline $\mathrm{CN}$ route, caud/deep vent/dors & $13 / 4 / 1(n=18)$ & $35 / 15 / 7(n=57)$ & 0.63 \\
\hline Tumor size in IAC (FN), $\mathrm{cm}^{*}$ & $1.01 \pm 0.46$ & $1.12 \pm 1.12$ & 0.66 \\
\hline Tumor size in IAC (CN), $\mathrm{cm}^{*}$ & $0.91 \pm 0.15(n=18)$ & $1.13 \pm 1.20(n=57)$ & 0.44 \\
\hline Tumor size in CPA (FN), $\mathrm{cm}^{*}$ & $2.24 \pm 1.04$ & $1.87 \pm 1.26$ & 0.22 \\
\hline Tumor size in CPA (CN), $\mathrm{cm}^{*}$ & $2.12 \pm 0.92(n=18)$ & $1.73 \pm 1.20(n=57)$ & 0.22 \\
\hline Vestibular symptom period, mos* & $37.0 \pm 16.3$ & $31.8 \pm 14.6$ & 0.16 \\
\hline Cochlear symptom period, mos ${ }^{*}$ & $18.4 \pm 19.6$ & $11.6 \pm 12.7$ & 0.13 \\
\hline Preop HB grade, I//I & $1 / 22$ & $4 / 62$ & $1.00 \dagger$ \\
\hline Preop GR hearing grade, I/II/III & $4 / 6 / 8(n=18)$ & $23 / 17 / 17(n=57)$ & 0.34 \\
\hline Extent of resection, GTR/NTR/STR/PR & $8 / 6 / 9 / 0$ & $10 / 31 / 23 / 2$ & 0.12 \\
\hline FN adhesion, mild/strong & $8 / 15$ & $33 / 33$ & 0.21 \\
\hline $\mathrm{CN}$ adhesion, mild/strong & $2 / 16(n=18)$ & $6 / 51(n=57)$ & $1.00 \dagger$ \\
\hline Initial stimulation (FN), mA* & $1.80 \pm 0.65$ & $1.85 \pm 0.54$ & 0.72 \\
\hline Degree of fanning (FN), mild/strong & $8 / 15$ & $25 / 41$ & 0.79 \\
\hline Degree of fanning (CN), mild/strong & $4 / 14(n=18)$ & $21 / 36(n=57)$ & $0.39 \dagger$ \\
\hline Degree of meatal filling $(\%)^{*}$ & $94.6 \pm 6.2$ & $95.2 \pm 5.3$ & 0.66 \\
\hline \multicolumn{4}{|c|}{$\begin{array}{l}\text { Caud = caudal; } C N=\text { cochlear nerve; com vent = common ventral; } C P A=\text { cerebellopontine angle; deep vent = deep ventral; dors = dorsal; FN } \\
=\text { facial nerve; GTR = gross-total removal; IAC = internal auditory canal; IVN = inferior vestibular nerve; } N T R=\text { near-total removal; } P R=\text { partia } \\
\text { removal; rost = rostromedial; STR = subtotal removal; SVN = superior vestibular nerve. } \\
\text { * Mean } \pm \text { SD. } \\
\text { † Fisher's exact test. }\end{array}$} \\
\hline
\end{tabular}


TABLE 2. Stepwise logistic regression analysis for factors associated with same-grade preservation of cochlear and facial nerve function*

\begin{tabular}{lccccc}
\hline \multicolumn{1}{c}{ Variable } & Coefficient & SE & Adjusted OR & $95 \% \mathrm{Cl}$ & $\mathrm{pValue}$ \\
\hline Cochlear nerve preservation & & & & & \\
\hline Final AEDNAP APR & -0.061 & 0.018 & 0.940 & $0.909-0.973$ & $<0.0001$ \\
\hline Group & -1.730 & 0.737 & 0.177 & $0.042-0.752$ & 0.019 \\
\hline Facial nerve preservation & & & & & \\
\hline$\quad$ Final orbicularis oris muscle FREMAP APR & -0.094 & 0.022 & 0.910 & $0.871-0.951$ & $<0.0001$ \\
\hline$\quad$ Group $\dagger$ & -1.685 & 0.728 & 0.186 & $0.045-0.772$ & 0.021 \\
\hline * Only statistically significant variables are presented in this table. & & & &
\end{tabular}

ing changes associated with surgical procedures. Neither the total number of injuries nor the total acute decline in function of both the cochlear nerve and facial nerve differed between the 2 treatment groups. However, the total waiting time, or duration of recuperation period, was significantly longer for patients in the extended recuperation treatment group. One-way analysis of covariance with acute decline as the covariate showed that the following were significantly smaller for patients in the extended recuperation treatment group: traction injury-related permanent loss in orbicularis oris muscle APR $(p=0.009)$, traction injury-related permanent loss in AEDNAP APR $(\mathrm{p}=0.018)$, and ultrasonic injury-related permanent loss in orbicularis oris muscle FREMAP APR $(p=0.015)$, although the AEDNAP APR and orbicularis oris muscle FREMAP APR for total permanent loss did not differ between the 2 groups (Table 3 ).

\section{Resection Rate and Tumor Recurrence}

Subtotal $(\geq 95 \%)$ tumor removal was achieved in 87 $(97.8 \%)$ of 89 patients. Gross-total or near-total removal was achieved in $14(60.9 \%)$ of 23 patients in the standard treatment group and in $41(61.2 \%)$ of 67 patients in the extended recuperation treatment group. To date, addition-

TABLE 3. Changes in monitoring related to the surgical procedure*

\begin{tabular}{|c|c|c|c|}
\hline \multirow[b]{2}{*}{ Variable } & \multicolumn{2}{|c|}{ Treatment Group } & \multirow[b]{2}{*}{$\mathrm{p}$ Value } \\
\hline & Standard & Extended Recuperation & \\
\hline Total waiting time (FN), mins & $1.3 \pm 6.3(n=23)$ & $11.8 \pm 21.7(n=66)$ & 0.001 \\
\hline Total waiting time $(\mathrm{CN})$, mins & $8.3 \pm 20.1(n=18)$ & $49.3 \pm 52.6(n=57)$ & $<0.0001$ \\
\hline Total AD in orbicularis oris muscle FREMAP APR, $\%$ & $17.4 \pm 21.3(n=23)$ & $17.7 \pm 29.4(n=66)$ & 0.97 \\
\hline Total AD in AEDNAP APR, \% & $52.9 \pm 41.0(n=18)$ & $61.1 \pm 48.9(n=57)$ & 0.53 \\
\hline Total PL in orbicularis oris muscle FREMAP APR, $\%$ & $12.8 \pm 17.8(n=23)$ & $11.2 \pm 11.2(n=66)$ & 0.74 \\
\hline Total PL in AEDNAP APR, \% & $50.1 \pm 40.5(n=18)$ & $36.8 \pm 32.2(n=57)$ & 0.16 \\
\hline No. of acute injuries (FN) & $0.7 \pm 0.9(n=23)$ & $0.5 \pm 0.8(n=66)$ & 0.25 \\
\hline No. of acute injuries (CN) & $1.6 \pm 1.0(n=18)$ & $1.4 \pm 1.0(n=57)$ & 0.34 \\
\hline $\begin{array}{l}\text { Traction injury-related PL in orbicularis oris muscle } \\
\text { FREMAP APR, \% }\end{array}$ & $9.49 \pm 1.02(n=23)$ & $6.33 \pm 0.60(n=66)$ & $0.009 \dagger$ \\
\hline Traction injury-related PL in AEDNAP APR, \% & $30.10 \pm 4.39(n=18)$ & $17.93 \pm 2.47(n=57)$ & $0.018 \dagger$ \\
\hline $\begin{array}{l}\text { Crush injury-related PL in orbicularis oris muscle } \\
\text { FREMAP APR, \% }\end{array}$ & $4.43 \pm 0.81(n=23)$ & $2.77 \pm 0.48(n=66)$ & $0.08 \dagger$ \\
\hline Crush injury-related PL in AEDNAP APR, \% & $7.94 \pm 0.94(n=18)$ & $7.41 \pm 0.53(n=57)$ & $0.62 \dagger$ \\
\hline $\begin{array}{l}\text { Thermal injury-related PL in orbicularis oris muscle } \\
\text { FREMAP APR, \% }\end{array}$ & $2.36 \pm 0.75(n=23)$ & $1.62 \pm 0.44(n=66)$ & $0.40 \dagger$ \\
\hline Thermal injury-related PL in AEDNAP APR, \% & $6.56 \pm 1.36(n=18)$ & $4.83 \pm 0.76(n=57)$ & $0.28 \dagger$ \\
\hline $\begin{array}{l}\text { Ultrasonic injury related PL in orbicularis oris muscle } \\
\text { FREMAP APR, \% }\end{array}$ & $4.56 \pm 0.70(n=23)$ & $2.53 \pm 0.41(n=66)$ & $0.015 \dagger$ \\
\hline Ultrasonic injury related PL in AEDNAP APR, \% & $7.26 \pm 1.28(n=18)$ & $5.16 \pm 0.72(n=57)$ & $0.16 \dagger$ \\
\hline Final stimulation (FN), $\mathrm{mA}$ & $2.00 \pm 0.84(n=23)$ & $2.02 \pm 0.61(n=66)$ & 0.93 \\
\hline Final orbicularis oris muscle FREMAP APR, \% & $59.2 \pm 20.8(n=23)$ & $78.5 \pm 18.8(n=66)$ & $<0.0001$ \\
\hline Final AEDNAP APR, $\%$ & $28.7 \pm 27.2(n=18)$ & $46.4 \pm 27.9(n=57)$ & 0.022 \\
\hline
\end{tabular}

$\mathrm{AD}=$ acute decline; $\mathrm{PL}=$ permanent loss

* Values are presented as the mean \pm SD (number).

$\dagger$ One-way analysis of covariance-adjusted means for acute decline. 
al resection for tumor recurrence has been needed by 1 (1.1\%) patient who had undergone partial tumor removal.

\section{Discussion}

Continuous direct AEDNAP and FREMAP monitoring during AN surgery is useful for preservation of function and enables real-time monitoring of the extent of damage to the cochlear and facial nerves. Conventional BAEP monitoring takes a small amount of time to detect waveform changes (wave-V loss), which is based on averaging of 500-2000 electrical signals, ${ }^{10-12,16,21,24}$ whereas continuous direct AEDNAP monitoring can recognize immediate risk for nerve damage. Our present method of continuous direct FREMAP monitoring uses our specially developed FREMAP electrode, in contrast to the semispherical, freely mobile electrode previously used to stimulate the cisternal portion of the facial nerve. ${ }^{2}$ We always place a rim around the electrode to ensure stable attachment to the facial nerve root, which enters the brainstem at the junction of the pons and medulla.

Previous studies have identified the following factors as favorable for preserving nerve function: tumor size less than $1.5 \mathrm{~cm}$ (Fig. 3A and B), low degree of meatal filling (Fig. 3A), tumor origin in the superior vestibular nerve, ${ }^{8,14}$ mild adhesion at the cochlear nerve-tumor interface (Fig. 3C and D), ${ }^{28}$ oligodendroglial myelin coverage at the cisternal segment of the cochlear nerve, ${ }^{20}$ maintenance of cochlear nerve microcirculation, ${ }^{25}$ and shorter absolute wave-V latency. ${ }^{6}$ Our study demonstrated that final AEDNAP APR and final orbicularis oris muscle FREMAP APR are also indicative of preserved nerve function. In addition, the period of recuperation could be crucial to preservation of nerve function. In a rat model, mechanical injuries to the cochlear nerve determined the extent of permanent loss of BAEP and cochlear nucleus action potentials, whereas recuperation treatment diminished nerve damage. ${ }^{22-24}$ These findings were experimentally confirmed in rat ischemic injury models, ${ }^{4}$ in pig thermal and ultrasonic injury models, and in human cadaveric specimens of the facial nerve and sciatic nerves. ${ }^{1,3,5,9,29}$

In our study, the only difference between the standard treatment group and the extended recuperation treatment group was the duration of recuperation. No significant differences in total acute decline and number of acute injury events were observed between the 2 groups. The final AEDNAP APR for the cochlear nerve $(46.4 \% \pm$ $27.9 \%$ vs $28.7 \% \pm 27.2 \%, \mathrm{p}=0.022$ ) and the final orbicularis oris muscle FREMAP APR for the facial nerve $(78.5 \% \pm 18.8 \%$ vs $59.2 \% \pm 20.8 \%, p<0.0001)$ were significantly higher for patients in the extended recuperation treatment group than for those in the standard treatment group (Table 3). However, extended recuperation treatment was not necessarily an effective method for preventing damage caused by every surgical procedure in this study. AN surgery consists of 10 steps and is considered to carry extreme risk for nerve damage. Previously, the procedures with the greatest risk for severe nerve damage were unclear. This study determined the optimal threshold for same-grade preservation of function from the ROC curves for patients in the standard treatment group. Our thresholds for AEDNAP APR of $36.5 \%$ and FREMAP APR of $61.5 \%$ provided indicators of when the neurosurgeon should temporarily stop the surgical procedure. Extended recuperation treatment at this time resulted in neurological recovery, especially after traction injury (Table 3). The sensory and motor nerves seemed to differ in potential for injury severity. Ultrasonic injury is a direct neurological insult but is less traumatic than crush or thermal injury. Extended recuperation treatment seemed effective after ultrasonic injury of the facial nerve. In contrast, ultrasonic injury caused severe damage to the cochlear nerve. Therefore, severe injuries such as crush and thermal injury should always be avoided, but extended recuperation treatment could help restore nerve damage, especially after traction injury.

The inevitable limitations of this retrospective and single-institution study include selection bias, observer bias (especially for postoperative facial function), a heterogeneous patient cohort, and absence of a direct comparison group (especially for the recuperation data). It is possible that recuperation time alone did not account for the improved outcome in the extended recuperation treatment group. It is also possible that surgical technique improved because of case volume and surgeon experience over the years, which could have contributed to improved outcomes. However, the same surgical strategy of extended recuperation treatment under continuous direct AEDNAP and FREMAP monitoring could be applicable to other cranial nerve neuroma surgery.

\section{Conclusions}

Outcomes after AN surgery can be improved by avoiding irreversible injury, such as crush and thermal injury, and by maximizing the recuperation period after reversible injury, such as traction injury, through use of the extended recuperation treatment strategy.

\section{Acknowledgments}

We thank Tomio Sasaki of Kyushu University Hospital for surgical expertise and the medical technologists at Toranomon Hospital, especially Husae Kawana, Satoko Anno, and Yuka Kimura, for neurophysiological monitoring advice.

\section{References}

1. Abbas GM, Jones RO: Measurements of drill-induced temperature change in the facial nerve during mastoid surgery: a cadaveric model using diamond burs. Ann Otol Rhinol Laryngol 110:867-870, 2001

2. Amano M, Kohno M, Nagata O, Taniguchi M, Sora S, Sato $\mathrm{H}$ : Intraoperative continuous monitoring of evoked facial nerve electromyograms in acoustic neuroma surgery. Acta Neurochir (Wien) 153:1059-1067, 2011

3. Anderson TP, Wakim KG, Herrick JF, Bennett WA, Krusen FH: An experimental study of the effects of ultrasonic energy on the lower part of the spinal cord and peripheral nerves. Arch Phys Med Rehabil 32:71-83, 1951

4. Asai Y, Umemura K, Nakashima M: Reversibility of compound action potential during the acute phase after transitory local ischemia. Ann Otol Rhinol Laryngol 105:472-475, 1996

5. Aslan A, Vatansever HS, Aslan GG, Eskiizmir G, Giray G: Effect of thermal energy produced by drilling on the facial 
nerve: histopathologic evaluation in guinea pigs. J Laryngol Otol 119:600-605, 2005

6. Brackmann DE, Owens RM, Friedman RA, Hitselberger WE, De la Cruz A, House JW, et al: Prognostic factors for hearing preservation in vestibular schwannoma surgery. Am J Otol 21:417-424, 2000

7. Esquia-Medina GN, Grayeli AB, Ferrary E, Tubach F, Bernat I, Zhang Z, et al: Do facial nerve displacement pattern and tumor adhesion influence the facial nerve outcome in vestibular schwannoma surgery? Otol Neurotol 30:392-397, 2009

8. Jacob A, Robinson LL Jr, Bortman JS, Yu L, Dodson EE, Welling DB: Nerve of origin, tumor size, hearing preservation, and facial nerve outcomes in 359 vestibular schwannoma resections at a tertiary care academic center. Laryngoscope 117:2087-2092, 2007

9. James JA, Dalton GA, Freundlich HF, Bullen MA, Wells PNT, Hughes DA, et al: Histological, thermal and biochemical effects of ultrasound on the labyrinth and temporal bone. Acta Otolaryngol 57:306-312, 1964

10. Legatt AD: Mechanisms of intraoperative brainstem auditory-evoked potential changes. J Clin Neurophysiol 19:396408, 2002

11. Matthies C, Samii M: Direct brainstem recording of auditory-evoked potentials during vestibular schwannoma resection: nuclear BAEP recording. Technical note and preliminary results. J Neurosurg 86:1057-1062, 1997

12. Matthies C, Samii M: Management of vestibular schwannomas (acoustic neuromas): the value of neurophysiology for intraoperative monitoring of auditory function in 200 cases. Neurosurgery 40:459-468, 1997

13. Matthies C, Samii M: Vestibular schwannomas and auditory function: options in large T3 and T4 tumors? Neurochirurgie 48:461-470, 2002

14. Mohr G, Sade B, Defour JJ, Rappaport JM: Preservation of hearing in patients undergoing microsurgery for vestibular schwannoma: degree of meatal filling. J Neurosurg 102:1-5, 2005

15. Moriyama T, Fukushima T, Asaoka K, Roche PH, Barrs DM, McElveen JT Jr: Hearing preservation in acoustic neuroma surgery: importance of adhesion between the cochlear nerve and the tumor. J Neurosurg 97:337-340, 2002

16. Neu M, Strauss C, Romstöck J, Bischoff B, Fahlbusch R: The prognostic value of intraoperative BAEP patterns in acoustic neurinoma surgery. Clin Neurophysiol 110:1935-1941, 1999

17. Romstöck J, Strauss C, Fahlbusch R: Continuous electromyography monitoring of motor cranial nerves during cerebellopontine angle surgery. J Neurosurg 93:586-593, 2000

18. Samii M, Matthies C: Management of 1000 vestibular schwannomas (acoustic neuromas): the facial nerve-preservation and restitution of function. Neurosurgery 40:684695, 1997

19. Sasaki T, Shono T, Hashiguchi K, Yoshida F, Suzuki SO: Histological considerations of the cleavage plane for preservation of facial and cochlear nerve functions in vestibular schwannoma surgery. Clinical article. J Neurosurg 110:648-655, 2009
20. Schefter RP, Harner SG: Histologic study of the vestibulocochlear nerve. Ann Otol Rhinol Laryngol 95:146-150, 1986

21. Schmerber S, Lavieille JP, Dumas G, Herve T: Intraoperative auditory monitoring in vestibular schwannoma surgery: new trends. Acta Otolaryngol 124:53-61, 2004

22. Sekiya T, Møller AR, Jannetta PJ: Pathophysiological mechanisms of intraoperative and postoperative hearing deficits in cerebellopontine angle surgery: an experimental study. Acta Neurochir (Wien) 81:142-151, 1986

23. Sekiya T, Okabe S, Iwabuchi T: Damage of the peripheral auditory system after operations in the cerebellopontine angle. A scanning electron-microscopic observation in dogs. Surg Neurol 30:117-124, 1988

24. Sekiya T, Shimamura N, Yagihashi A, Suzuki S: Axonal injury in auditory nerve observed in reversible latency changes of brainstem auditory-evoked potentials (BAEP) during cerebellopontine angle manipulations in rats. Hear Res 173: 91-99, 2002

25. Strauss C, Bischoff B, Neu M, Berg M, Fahlbusch R, Romstöck J: Vasoactive treatment for hearing preservation in acoustic neuroma surgery. J Neurosurg 95:771-777, 2001

26. Strauss C, Romstöck J, Fahlbusch R, Rampp S, Scheller C: Preservation of facial nerve function after postoperative vasoactive treatment in vestibular schwannoma surgery. Neurosurgery 59:577-584, 2006

27. Tomii M, Onoue H, Yasue M, Tokudome S, Abe T: Microscopic measurement of the facial nerve root exit zone from central glial myelin to peripheral Schwann cell myelin. J Neurosurg 99:121-124, 2003

28. Wanibuchi M, Fukushima T, McElveen JT Jr, Friedman AH: Hearing preservation in surgery for large vestibular schwannomas. Clinical article. J Neurosurg 111:845-854, 2009

29. Young W, Cohen AR, Hunt CD, Ransohoff J: Acute physiological effects of ultrasonic vibrations on nervous tissue. Neurosurgery 8:689-694, 1981

30. Zaouche S, Ionescu E, Dubreuil C, Ferber-Viart C: Pre- and intraoperative predictive factors of facial palsy in vestibular schwannoma surgery. Acta Otolaryngol 125:363-369, 2005

\section{Author Contributions}

Conception and design: Nakatomi, Miyazaki. Acquisition of data: Nakatomi, Miyazaki, Kin, Yoshino, Usui. Analysis and interpretation of data: Nakatomi, Miyazaki, Tanaka, Oyama, Moriyama. Drafting the article: Nakatomi. Critically revising the article: all authors. Reviewed submitted version of manuscript: all authors. Approved the final version of the manuscript on behalf of all authors: Nakatomi. Statistical analysis: Nakatomi. Administrative/technical/material support: Nakatomi. Study supervision: Nakatomi.

\section{Correspondence}

Hirofumi Nakatomi, Department of Neurosurgery, The University of Tokyo Hospital, 7-3-1 Hongo, Bunkyo-ku, Tokyo 113-8655, Japan. email: hnakatomi-tky@umin.ac.jp. 